\title{
Studies on Chitosan, Chitin and Chitooligosaccharides and Their Biomedical Properties
}

\author{
Alef IBRAM \\ Romvac Company S.A., România
}

\begin{abstract}
Research in healthcare involves the use of natural resources in the manufacture of pharmaceutical products. Chito-oligosaccharides (CHOS) are described as homo- or heterooligomers of $\mathrm{N}$-acetylglucosamine and $\mathrm{D}$ glucosamine. As a starting material for CHOS production chitin or chitosan can be used. When it comes to the medical field, there are indications that CHOS may be developed and used as drugs against asthma, antibacterial agents, ingredients in wound-dressings and vectors in gene-therapy. The methods of production can rely on enzymatic conversions, chemical methods or combinations of these methods. There is ample literature concerning the biological effects of chitosans, but relatively little is known about the effects of CHOS, and the mechanisms behind observed bioactivities are generally poorly understood. This review is focused on the characterization of chitin and chitosan by presenting the biological properties, and on the enzymatic production of CHOS, and on further separation and purification methods for producing well-defined mixtures and also provides an overview of some of the most promising applications of CHOS.
\end{abstract}

Keywords: chitooligosaccharide (CHOS); chitosan; biological properties; biomedical uses; chitin

\section{Introduction}

A major interest in modern medicine is represented by the biometerials with marine origins. Among these, chitin and chitosan received special attention in the medical fields due to their unique properties.

Chitin and its deacetylated derivative, chitosan are natural polymers composed of randomly distributed $\beta$-(1-4)-linked D-glucosamine (deacetylated unit) and N-acetylD-glucosamine (acetylated unit). Both chitin and chitosan cannot be defined as a unique chemical structure, but as a family of polymers, due to their natural origin, and also present a high variability in their chemical and physical properties. This variability is related not only to the origin of the samples but also to their method of 
preparation. So, a complete characterization of the samples is necessary (Sirbu R., 2019).

Since the oligosaccharides have several interesting bioactivities, production of welldefined chito-oligosacharides (CHOS)-mixtures, or even pure CHOS, is of great interest. Understanding the mechanisms underlying these bioactivities is of major importance. It could be noted that some of the biological effects reported for chitosan in fact may be due to CHOS, which emerge when chitosan is degraded by naturally occurring hydrolytic enzymes (Berit B. Aam, 2010).

Many studies and research have been made concerning the biological effects of chitosans, but relatively little is known about the effects of chito-oligosaccharides (CHOS) (Kim S.-K., 2005). Initially it was thought that CHOS need to interact with proteins that either act on chitin (chitinases), or that are supposed to bind to chitin forms and detect the biopolymer (You Y., 2004).

In the pharmaceutical and medical fields, CHOS may be used and developed as ingredients in wound-dressings (Ribeiro M.P., 2009), drugs against asthma, antibacterial agents (Elias J.A., 2005). According to other research CHOS may also be used to reduce metastasis and tumor-growth in cancer, increase bone-strength in osteoporosis, could prevent malaria by inhibiting chitinases in Plasmodium parasites, CHOS help lower the levels of glucose in diabetics, had have immune modulatory effects and anti-fungal activities.

It is thought that the majority of the biological effects of chitosan are in fact due to CHOS. These effects emerge when chitosan is degraded by naturally occurring hydrolytic enzymes.

\section{Characterization of Chitin, Chitosan and Chito-Oligosacharides (Chos)}

Chitin is a natural polymer, a linear polysaccharide consisting of $\beta(1 \rightarrow 4)$ linked $N$ acetyl-D-glucosamine (Fig.1). After cellulose, chitin is the second most abundant polysaccharide in nature. It is present in the cell walls of fungi and yeasts and in the exoskeletons of insects and arthropods such crabs, lobsters and shrimps. In reference to the physico-chemical properties it can be noted that chitin is insoluble in water and exists mainly in two crystalline polymorphic forms, $\alpha$ and $\beta . \alpha$-chitin is found in the exoskeleton of arthropods, in insects and in fungal and yeast cell walls. $\beta$-chitin occurs less frequently in nature than $\alpha$-chitin, but can be extracted from squid pens (Mustafa A., 2015). 


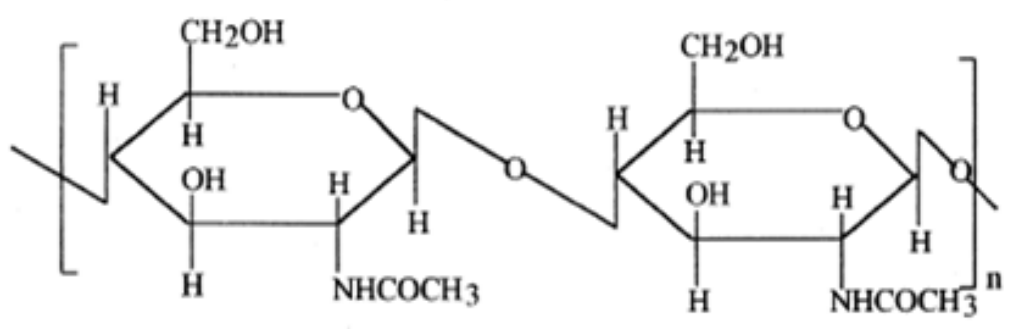

Chitin

Fig.1. Structure of chitin

Chitosan is the natural derivative of chitin and it can be prepared by partial deacetylation, and is a heteropolymer of $\beta(1 \rightarrow 4)$ linked $N$-acetyl- $D$-glucosamine and D-glucosamine (Fig.2). In comparation with chitin, chitosan is soluble in dilute aqueous acid solutions. Chitosan can also be described as a continuum of soluble polymeric chitin derivatives. These polymeric chitin derivatives can be characterized according to the fraction of $N$-acetylated residues $\left(\mathrm{F}_{\mathrm{A}}\right)$ or degree of $N$-acetylation (DA), the degree of polymerization (DP) or the molecular weight $\left(\mathrm{M}_{\mathrm{W}}\right)$, the molecular weight distribution (PD, for poly-dispersity), and the pattern of $N$-acetylation (PA) or sequence (Bhatnagar, A., 2009). Due to its remarkable physic-chemical and biological properties such as non-toxicity, biocompatibility and biodegradability, chitosan is used in numerous applications and fields (pharmacy, medicine, agriculture, cosmetics, water treatment) (Kim S.-K., 2005; Vårum K.M., 2005).

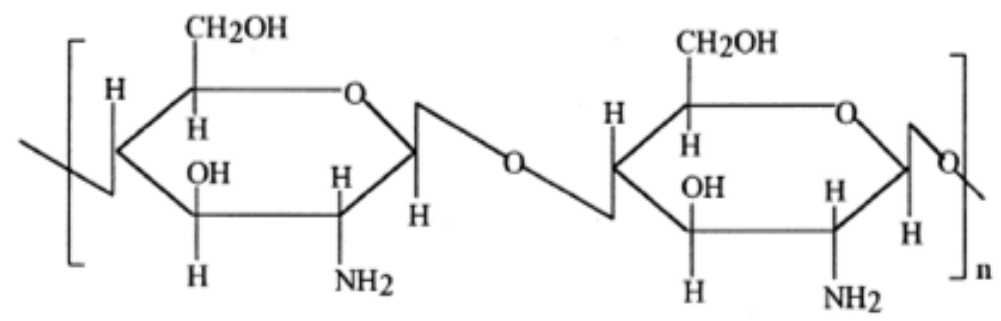

Chitosan

Fig.2. Structure of chitosan

Due to their natural origin, both chitin and chitosan are defined as a family of polymers which present a high variability in their chemical and biological properties such as biocompatibility, biodegradability, mucoadhesion, anticholesterolemic, antitumoral, hemostatic and antimicrobial effect (Sirbu R., 2019).

The main parameters that affect the polymer properties are DD (degree of deacetylation), Mw (molecular weight), polydispersity and crystalinity. 
The purity (ash content), the moisture, the content of heavy metals, endotoxin and proteins must be determined for applications related to human consumption such as food and medical applications.

It has been demonstrated that the DD is one of the most important chemical characteristics, which could influence the performance of chitosan in many of its applications. The influence of average Mw on the viscosity development of aqueos solutions plays a significant role in the biochemical and biopharmacological significance of chitosan (Tharanathan R.N., 2003). Due to its low solubility chitin Mw is not easily determined. Table 1 . shows the various methods for the determination of chitin and chitosan characteristics (Inmaculada Aranaz, 2009).

Table 1. Physicochemical Characteristics of Chitin and Chitosan and the Determination Methods

\begin{tabular}{|l|l|}
\hline Physicochemical Characteristics & Determination Method \\
\hline DD & $\begin{array}{l}\text { Infrared Spectroscopy } \\
\text { UV- spectrophotometry } \\
\text { Nuclear magnetic resonance spectroscopy } \\
\text { Potentiometric titration }\end{array}$ \\
\hline $\begin{array}{l}\text { Average Mw and/or Mw } \\
\text { distribution }\end{array}$ & $\begin{array}{l}\text { Viscosimetry } \\
\text { Gel Permeation cromatography }\end{array}$ \\
\hline Moisture content & Gravimetric analysis \\
\hline Ash content & Gravimetric analysis \\
\hline Protein & Bradford method \\
\hline
\end{tabular}

As is shown in Table.1 different result are obtained when using methods based on different principles. In present, even the best characterized chitosans available on the market are usually described olny with regard to their average degree of acetylation and their average degree of polymerization (DP), their ash content and the absence of contaminating bacteria (Moerschbacher B., 2007).

Apart from the specific characteristics, which are specific fosr each application, there is a degree of consensus regarding general characteristics that must be present in chitosan samples to be used in the field of biomedical applications: moisture content $\%$, ash content $\%$, protein content $\%$, insolubility $\%$, turbidity NTU units, DD $\%$. For pharmaceutical applications, the chitosan requirements are: colour: white or slight yellow, particle size $<0.3 \mathrm{~mm}$, density $1.35-1.40 \mathrm{~g} / \mathrm{cm}^{3}$, pH 6.5-7.5 (Sirbu R., 2019).

Chitin and chitosan are currently receiving a great deal of interest in the medical and pharmaceutical applications due to their interesting properties that make them suitable for use in the biomedical field, such as biocompatibility, biodegradability and non toxicity. Other properties such as analgesic, antitumor, hemostatic, 
hypocholesterolemic, antimicrobian and antioxidant properties have also been reported. A better understanding of the mechanism of these properties makes it necessary for chitosan to be well characterized and purified from accompanying compounds (Muzzarelli R.A.A, 2005). In addition chitin and chitosans derivatized in a variety of fashions can be used to prove molecular hypothesis for the biological activity. The parameter with a higher effect is the DD, because the majority of the biological properties are related to the cationic behaviour of the chitosan. In some cases, the Mw has a predominant role. Beside the DD and Mw, other properties such as chain conformation, solubility or degree of substitution have been studied. Chitosans produced by heterogenous deacetylation, with a block arrangement of acetylated units, have a tendency to form aggregates in aqueous solutions. Table. 2 shows the relationship between some chitin and chitosan biological properties and their physicochemical characteristics (Inmaculada Aranaz, 2009).

Table 2 . The relationship between some chitin and chitosan biological properties and their physicochemical charateristics

\begin{tabular}{|l|l|}
\hline Property & Characteristic \\
\hline Biodegradability & DD, distribution of acetyl groups Mw \\
\hline Biocompatibility & DD \\
\hline Mucoadhesion & DD, Mw (only chitosan) \\
\hline Hemostatic effect & DD, Mw \\
\hline Analgesic effect & DD \\
\hline Adsorbtion enhancer & DD (only chitosan) \\
\hline Antimicrobian effect & Mw \\
\hline Anticolesterolemic effect & DD, Mw, viscozity \\
\hline Antioxidant effect & DD,Mw \\
\hline
\end{tabular}

\section{Biological Properties of Chitosan Biodegradability}

Chitin and chitosan are absent from mammals but they can be degraded in vivo by several proteases (lysozyme, papain, pepsin). Their biodegradation leads to the release of non-toxic oligosaccharides of variable length which can be subsenquently incorporated to glycosaminoglycans and glycoproteins, to metabolic pathways or be excreted. A degradation role on chitin and chitosan seems to play a non-specific protease present in all mammalian tissues- lysozyme. The lengths of the chains $(\mathrm{Mw})$ affects the degradation rate (Huang M., 2004). The understanding and control of the degradation rate of chitin and chitosan-based devices is of great interest since degradation is essential in many small and large molecule releaseapplications and in functional tissue regeneration applications. Ideally, the rate of scaffold degradation should mirror the rate of new tissue formation or be adequate for the controlled release of bioactive molecules (Mustafa A., 2016). Thus, it is important to understand and control both the mechanism and the rate by which each material is degraded. The degradation rate also affects the biocompatibility since very fast rates of degradation 
will produce an accumulation of the amino sugars and produce an inflammatory response.

Chitosan samples with low DD induce an acute inflammatory response while chitosan samples with high DD induce a minimal response due to the low degradation rate. Degradation has been shown to increase as DD decreases. Kofuji et al. (2005) investigated the enzymatic behaviours of various chitosans by observing changes in the viscosity of chitosan solution in the presence of lysozyme (Kofuji K., 2005). They found that chitosan with a low DD tended to be degraded more rapidly. It can be concluded that it is impossible to estimate biodegradation rate from the DD alone.

\section{Biocompatibility}

Both chitin and chitosan show very good compatibility but this property depends on the characteristics of the sample (natural source, method of preparation, Mw and DD). Due to its higher versatility and biological properties the majority of the assays have been carried out on chitosan samples. Although the gastrointestinal enzymes can partially degrade both chitin and chitosan, when both polymers are orally administered they are not absorbed. For this reason, they are considered as not bioavailable. Toxicity of chitosan is reported to depend on DD. It was reported that chitosans with DD higher than 35\% showed low toxicity, while a DD under 35\% caused dose dependant toxicity. On the other hand, Mw of chitosan did not influence toxicity. Residual proteins in chitin and chitosan could cause allergic reactions such as hypersensitivity. The protein content in a sample depends on the source of the sample and, especially, on the method of preparation (Mustafa A., 2015).

\section{Hemostatic effect}

Chitosan presents anticoagulant activity tested in vitro (Park P.J, 2004). The anticoagulant activity of chitosan seems to be related to its positive charge since red blood cells' membranes are negatively charged and chitin is less effective than chitosan. The hemostatic effect of chitosan is not related to the clasic coaulation pathways, but it can promote platelet agregation. The blood platelets play a very important role in the coagulation process and can lead to hemostatis and thrombosis. Besides platelets and erythrocytes, chitosan also accelerates thrombin generation.

\section{Analgezic effect}

It was reported that both chitin and chitosan show analgesic effect. The analgesic effect of these biopolymers on inflammatory pain has been studied due to intraperitoneal administration of acetic acid. Chitosan showed a greater effect than chitin. This difference is due to the different action mechanism of the two polymers. It was demonstrated that the main analgesic effect of chitosan is the absorption of proton ions released in the inflammatory area (Inmaculada Aranaz, 2009). 


\section{Antitumor activity}

An antitumor activity of chitosan has been claimed by inhibition of the growth of tumor cells mainly due to an immunostimulation effect. chitosan oligomers possess antitumor activities tested both in vitro and in vivo (Jeon Y.J, 2002).

Studies carried out using mice that had ingested low-Mw chitosan revealed significant antimetastatic effects of chitosan against Lewis lung carcinoma. Partially deacetylated chitin as well as chitin with a carboxymethyl group have also been effective to demote tumor progression. The suggested mechanism involves immunostimulating effects of chitin and its carboxymethyl derivatives via stimulation of cytolytic T-lymphocytes. This activity increases with smaller molecular sizes and it is suggested that they have immunostimulating effects that activate peritoneal macrophages and stimulate nonspecific host resistance. However, higher $\mathrm{Mw}$ oligomers have also exhibited antitumor activity. The effect of chitosan on tumor growth and metastasis was studied. The activation of macrophages by chitosan is suggested to mediate its antitumor effects in vivo, while its angiogenic inducing properties may be the harmful effects of chitosan, such as promotion of tumor growth and invasion (Ueno H., 2001).

\section{Anticholesterolemic effect}

There are several proposed mechanisms for cholesterol reduction by chitosan. The entrapment caused by a viscous polysaccharide solution is thought to reduce the absorption of fat and cholesterol in the diet. On the other hand,the presence of the amino group in its structure determines the electrostatic force between chitosan and anion substances, such as fatty acids and bile acids. The interaction between chitosan and anionic surfaceactive materials (phospholipids, bile acids) depends on its three types of reactive functional groups: the amino group at the $\mathrm{C} 2$ position and primary and secondary hydroxyl groups at the C-3 and C-6 positions, respectively. Although great effort has been made to find a correlation between the physicochemical characteristics of chitosan and its fat-binding capacity, only some significant relationships have been demonstrated (Inmaculada Aranaz, 2009).

\section{Antimicrobial activity}

The antimicrobial activity of chitin, chitosan, and their derivatives against different groups of microorganisms, such as bacteria, yeast, and fungi, has received considerable attention in recent years (Sirbu R., 2019). Two main mechanisms have been suggested as the cause of the inhibition of microbial cells bychitosan. The first mechanism refers to the interaction with anionic groups on the cell surface. Due to its polycationic nature, it causes the formation of an impermeable layer around the cell, which prevents the transport of essential solutes. Electron microscopy demostrated that the site of action is the outer membrane of gram negative bacteria. The permeabilizing effect has been observed at slightly acidic conditions in which chitosan is protonated, but this permeabilizing effect of chitosan is reversible (Helander I., 2001). The second mechanism involves the inhibition of the RNA and 
protein synthesis by permeation into the cell nucleus. In this case the $\mathrm{Mw}$ is the decisive property (Liu X., 2001). Other mechanisms have also been proposed. Chitosanmay inhibit microbial growth by acting as a chelating agent rendering metals, trace elements or essential nutrients unavailable for the organism to grow at the normal rate. Chitosan is also able to interact with flocculate proteins, but this action is highly pH-dependent.

\section{Antioxidative activity}

Chitosan has shown a significant scavenging capacity against different radical species, the results being comparable to those obtained with commercial antioxidants. Samples prepared from crab shell chitin with DD of 90,75 and $50 \%$ where evaluated on the basis of their abilities to scavenge 1,1-diphenyl-2-picrylhydrazyl (DPPH) radical, hydroxyl radical, superoxide radical and alkyl radical. The results revealed that chitosan with higher DD exhibited the highest scavenging activity (Park P.J., 2004). Chitosans of different size as well as their sulphate derivatives were assayed against superoxide and hydroxyl radicals. A negative correlation was found between chitosan Mw and activity. The chitosan sulphated derivatives presented a stronger scavenging effect on peroxide radicals but the chitosan of lowest Mw showed more considerable ferrous ion-chelating potency than others (Xing R., 2005). The chelation of metal ions is one of the reasons why chitosan may be considered as a potential natural antioxidant for stabilizing lipid containing foods to prolong shelf life. Chitosans may retard lipid oxidation by chelating ferrous ions present in the system, thus eliminating their prooxidant activity or their conversion to ferric ion.

Chito-oligosaccharides (CHOS) are oligomers prepared from chitosan by chemical or enzymatical methods. CHOS can be obtained starting from chitosan by acid hydrolysis or by enzymatic hydrolysis using chitinases or chitosanases. In result, the $\mathrm{F}_{\mathrm{A}}, \mathrm{M}_{\mathrm{W}}, \mathrm{PD}$ and $\mathrm{P}_{\mathrm{A}}$ of the resulting CHOS-mixture depend on the chitosan and the specificity of the enzyme used. It can be necessary, for certain compounds to enrich the product mixtures, and for this process the chitosan-enzyme combination is optimized (Berit B. Aam, 2010).

A recent statistic estimated the annual production of chitin in nature to be approximately 1010-1011 tons per year (Berit B. Aam, 2010). It was calculated that in 2007, the amounts of chitin, chitosan and their derivatives that are used and produced in industrial processes (Sannan T. Kurita, 1976) have been estimated to be about 30000 metric tons for chitin and about 10,000 metric tons for chitosan. It was suggested that it is a possibility to isolate chitosan directly from the cell walls of certain fungi, but chitosans that are commercially available are usually prepared from chitin by a heterogeneous deacetylation process (Sandford P.A., 2002).

There are two fundamentally different methods to prepare chitosans from chitin: homogeneous and heterogeneous deacetylation (Sannan T. Kurita, 1976). The homogeneous deacetylation process requires the chitin to be dissolved in an alkali solution during the deacetylation process (this needs low temperature and excessive 
stirring). The heterogeneous deacetylation process is a actually a two-phase process, in which the chitin is kept insoluble in a hot alkali solution. In order to facilitate the depolymerization to take place, some specific enzymes that act on chitin and chitosan are required: Chitinases and chitosanases, Human chitinases and Lysozyme.

\section{Chito-Oligosacharides (Chos) Production}

The production of CHOS is made bytwomethods: enzymaticandchemical.

\section{Enzymatic methods}

The research of this methods is poor in concrete results and cannot yet be considered an alternative to the chemical methods that are used in the present. In theory, chitin deacetylases could be used to produce chitosan (Tokuyasu K., 2000; Tsigos I., 2000) by hydrolyzing the $\mathrm{N}$-acetyl linkage and convert chitin to chitosan, but the insolubility and crystallinity of the chitin substrate makes it very difficult. Recent studies with chitinases have related that kinetics of the degradation reactions change considerably during the hydrolysis process. The enzymes that are used for this processes have very different binding affinities for different sequences on the substrate, leading to reactions that present multiphasic kinetics, resulting very different product mixtures. The other important issue to take into hand is processivity, because the degradation process may change during a reaction. It can stat from processive hydrolysis of polymeric chains and lead to non-processive hydrolysis of intermediate products due to the exhaustion of the polymeric material. We can conclude that the outcome of the enzymatic conversion can be influenced by choosing the right starting chitosan, the enzyme and the processing time (Sikorski P., 2005).

\section{Chemical Methods}

\subsection{Acid Hydrolysis of Chitosan}

One of the best known chemical methods for hydrolysis of chitosan is acid hydrolysis (Einbu A., 2007). The first studies on acid hydrolysis of chitosans had demonstrated that it is possible to convert fully deacetylated chitosan to CHOS in concentrated hydrochloric acid (Berit B. Aam, 2010). In later studies (Einbu A., 2007), using a variety of chitosans, the acid-catalyzed degradation rates of chitosans were shown to depend on $\mathrm{F}_{\mathrm{A}}$ (fraction of $\mathrm{N}$-acetylated residues), and the initial degradation rate constant was found to increase in direct proportion to $\mathrm{F}_{\mathrm{A}}$.

\subsection{Chemical Synthesis of Chito-Oligosacharides(Chos)}

The chemical synthesis of CHOS does not represent a frequent procedure in the present days, because it requires multiple protection and deprotection steps. Although chemical synthesis of CHOS results in pure compounds this method is time consuming and requires extensive use of organic solvents. In the speciality literature there are only a few examples of chemically synthesized CHOS. One example is that Kuyama et al. performed synthesis of fully deacetylated chitosan dodecamers starting with glucosamine monomers using phthalimido as the amino protective group. 
Another one, Aly et al. reported a method for synthesis of fully $\mathrm{N}$-acetylated CHOS from GlcN monomers using dimethylmaleoyl as an amino protective group for synthesis of chitotetraose and chitohexaose. The removal of the amino protective group and $N$-acetylation was performed in a one-pot reaction in order to result in the desired products (Berit B. Aam, 2010). In theory it can be a possibility to combine the two described protection methods in order to synthesize partly deacetylated CHOS, but this has so far not been reported in the scientific literature.

\section{Chito-oligosacharides(CHOS) purification}

It is known that enzymatically or chemically produced CHOS is usually composed of a mixture of oligomers differing in DP (degree of polymerization), $\mathrm{F}_{\mathrm{A}}$ (fraction of $\mathrm{N}$ acetylated residues) and $\mathrm{P}_{\mathrm{A}}$ (pattern of $N$-acetylation). Over the years, a multitude of techniques for separation and purification of CHOS have been reported, such as: gelfiltration, ultra-filtration, and ion exchange and metal affinity (Berit B. Aam, 2010) chromatography. In almost all cases is preferred to use a combination of these techniques in order to obtain homogenous CHOS fractions. All in all, as shown above, the production of pure CHOS fractions is generally a time consuming, expensive and challenging task.

\section{Pharmaceutical and biomedical properties of CHOS}

As shown above, due to its important physico-chemical properties and based on the fact that

it is a natural biopolymer, CHOS have a remarkable wide range of pharmaceutical and biomedical properties and activities.

\section{Applications of chitosan/CHOS in wound-dressings}

There are a multitude of studies that had very promissing and positive effects of chitosan used in wound-dressing (Mustafa A., 2015). Other studies developed in parallel on the same actions of CHOS had also very positive effects, in fact it was shown that the use of CHOS accelerates the wound healing process (You Y., 2004). In contact with the wound chitosan is likely to convert into CHOS by the action the the naturally occurring enzymes. This wound-healing effect that was presented initially for chitosan can be in fact the effect of CHOS converted by enzymes. The resulting conclusion is that the use of CHOS directly is more advantageous in wound dressing than chitosan, because of the more immediate effect.

Recent studies showed that hemostatic effects may also contribute to the beneficial effects of chitosan/CHOS in wound dressings. It was demonstrated that chitosan enhances platelet adhesion and aggregation and increases the release of the platelet derived growth factor-AB (PDGF-AB) and the transforming growth factor- $\beta 1$ (TGF$\beta 1$ ) from platelets in canine blood (Berit B. Aam, 2010). These two factors retract inflammatory cells which are important in wound healing. 


\section{Tumor growth inhibition}

In the 1970s it has been demonstrated that CHOS have anti-tumor effects, and reduce metastasis from tumors (Mustafa A., 2016). Initially it was believed that the antitumor activity was due to the cationic properties of $\mathrm{CHOS}$, and later, the $\mathrm{M}_{\mathrm{W}}$ was discovered to play a major role. Two researchers, Maeda and Kimura (Berit B. Aam, 2010) found that CHOS enhanced the natural killer activity in intestinal intraepithelial lymphocytes at the same time as they reduced tumor growth in mice, and suggested that this CHOS-activation of intestinal immune functions could be useful in treating tumors.

In recent years, the hypothesis that the anti-tumor effects of CHOS are related to their inhibitory effects on angiogenesis has received some attention. Angiogenesis is the formation of new capillary blood vessels from already existing blood vessels. This process is important for tumor formation, since tumor growth and metastasis require angiogenesis when the tumor reaches a certain size.

\section{Asthma}

AMCase is induced during $\mathrm{T}_{\mathrm{H}} 2$ inflammation through an interleukin (IL)-13 dependent mechanism. This was shown to be over-expressed in human asthmatic tissue (Elias J.A., 2005). Inhibition of the AMCase with the chitinase inhibitor reduced the inflammation (Berit B. Aam, 2010; Mustafa A., 2015). The fact that chitinases are a factor in host antiparasite responses and in asthmatic $\mathrm{T}_{\mathrm{H}} 2$ inflammation led to the hypothesis that asthma may be a parasite-independent antiparasite response (Elias J.A., 2005). In some studies it has been suggested that partially deacetylated CHOS can function as inhibitors of family 18 chitinases. So, we can conclude that CHOS have a great potential in use as an anti-inflammatory drug in patients with asthma.

\section{Increased bone strength}

The bone marrow is formed from the mesenchymal stem cells that are able to differentiate into chondrocytes, adipocytes and osteoblasts. Bone-tissue is mainly formed from bone matrix and osteoblasts (Mustafa A., 2015; Mustafa A., 2016). CHOS and chitosan are able to increase the differentiation of mesenchymal stem cells to osteoblasts and to facilitate the formation of bone-tissue. CHOS can increase calcium depositation in bone, because it is known that the mineralization process and bone strength are dependent on $\mathrm{Ca}^{2+}$ (Berit B. Aam, 2010).

\section{Conclusion}

Chitin, chitosan and CHOS have a wide spectrum of possible bioactivities, due to their remarkable properties. Although they are very versatile polymers and have promising bioactivities, the research must be continued and completed with welldefined CHOS preparations. Recent techniques for further purification of CHOS and for sequence determination are available, but are still quite challenging to exploit. Another challenge in the process of purification of CHOS is to find an economically 
acceptable solution. From this point of view, in the present it is cheaper to produce CHOS mixtures that require enriching for a specific bioactivity, rather than producing a pure compound form the beginning.

The vision for the future in medical health of these studies and researches is to successfully convert the abundant bio-resource that is chitin into CHOS-based medicines and pharmaceutical forms.

\section{References}

[1] Berit B. Aam, Ellinor B. Heggset, Anne Line Norberg, Morten Sørlie, Kjell M. Vårum, Vincent G. H. Eijsink, (2010). Production of Chitooligosaccharides and Their Potential Applications in Medicine, Mar. Drugs, 8, 1482-1517.

[2] Bhatnagar, A.; Sillanpää, M., (2009). Applications of chitin- and chitosanderivatives for the detoxification of water and wastewater - A short review. Adv.Colloid Interface Sci., 152, 26-38.

[3] Einbu, A.; Vårum, K.M., (2007). Depolymerization and de-N-acetylation of chitinoligomers in hydrochloric acid. Biomacromolecules, , 8, 309-314.

[4] Elias, J.A.; Homer, R.J.; Hamid, Q.; Lee, C.G., (2005). Chitinases and chitinaselike proteins in $\mathrm{T}_{\mathrm{H}} 2$ inflammation and asthma. J. Allergy Clin. Immunol., 116, 497-500.

[5] Helander I, Nurmiaho-Lassila E, Ahvenainen R, Rhoades J, Roller S., (2001). Chitosan disrupts the barrier properties of the outer membrane of Gramnegative Bacteria,Int J Food Microbiol, 71, 235-244.

[6] Huang M, Khor E, Lim L., (2004). Uptake and cytotoxicity of chitosan molecules and nanoparticles: effects of molecular weight and degree of deacetylation,Pharm Res., 21(2), 344-353.

[7] Inmaculada Aranaz, Marian Mengibar, Ruth Harris, (2009). Functional Characterization of Chitin and Chitosan, Current Chem Biol ,3, 203-230

[8] Jeon Y.J, Kim S.K., (2002). Antitumor activity of chitosan oligosaccharides produced in ultrafiltration membrance reactor system,J Microbiol Biotechnol, ,12(3), 503-507.

[9] Kim, S.-K., Rajapakse, N., (2005). Enzymatic production and biological activities of chitosan oligosaccharides (COS): A review. Carbohydr. Polym., 62, 357- 368.

[10] Kofuji K, Qian CJ, Nishimura M, Sugiyama I, Murata Y, Kawashima S., (2005). Relationship between physicochemical characteristics and functional properties of Chitosan,Eur Polym J, 41(11), 2784-2791.

[11] Liu X, Yun L, Dong Z, Zhi L, Kang D, (2001). Antibacterial action of chitosan and carboxymethylated chitosan,J Appl Polym Sci, 79(7), 1324-1335.

[12] Moerschbacher B, El Gueddari N., (2007). Bio-activity matrices for partially acetylated chitosan oligomers. Advances in chitin Science, IX (CD),10-23.

[13] Mustafa A., Cadar E., Sîrbu R., (2015). Pharmaceutical Uses of Chitosan in the Medical Field, Europ. J. Interdiscip. St., 3(1),35-40, 
[14] Mustafa A., Tomescu A., Cadar E., Cherim M., Sîrbu R., (2016). Polyelectrolyte Complexes Based On Chitosan And Natural Polymers, Europ. J.Interdiscip. St.,2 (1), 100-107.

[15] Muzzarelli R.A.A, Muzzarelli C., (2005). Chitosan chemistry: Relevance to the biomedical sciences, Adv Polym Sci., 186, 151-209.

[16] Park P. J, Je J. Y, Jung WK, Ahn C. B, Kim S. K., (2004). Anticoagulant activity of heterochitosans and their oligosaccharide sulfates,Eur Food Res Technol., 219, 529-33.

[17] Park P.J, Je J.Y, Kim S.K., (2004). Free radical scavenging activities of differently deacetylated chitosans using an ESR spectrometer, Carbohydr Polym, 55(1),17-22.

[18] Ribeiro, M.P., Espiga, A., Silva, D., Baptista, P., Henriques, J., Ferreira, C., Silva, J.C., Borges, J.P., Pires, E., Chaves, P., Correia, I.J., (2009). Development of a new chitosan hydrogel for wound dressing. Wound Repair Regen., 17, 817-824.

[19] Sandford, P.A., (2002). Commercial sources of chitin \& chitosan and their utilization. Advances in Chitin Science; Vårum, K.M., Domard, A., Smidsrød, O., Eds. Tapir: Trondheim, Norway, VI.

[20] Sannan, T. Kurita, K. Iwakura, Y., (1976). Studies on Chitin, 2. Effect of deacetylation on solubility. Macromol. Chem., 177, 3589-3600.

[21] Sikorski, P., Stokke, B.T., Sørbotten, A., Vårum, K.M., Horn, S.J., Eijsink, V.G., (2005). Development and application of a model for chitosan hydrolysis by a family 18 chitinase. Biopolymers, 77, 273-285.

[22] Sirbu R., Mustafa A., Tomescu A., Stanciu G., Cadar E., (2019). Rheological and Microbiological Study on Bio-Composites with Marine Chitosan Polymers from Black Sea Stone Crabs used in Medical Therapy of Tissue Regeneration, Rev. Materiale Plastice, , 56(1), 148-155,.

[23] Tharanathan RN, Kittur F. S., (2003). Chitin: The Undisputed Biomecule of Great Potential. Crit Rev Food Sci Nut., 43(1), 61-87.

[24] Tokuyasu, K., Mitsutomi, M., Yamaguchi, I., Hayashi, K., Mori, Y., (2000). Recognition of chitooligosaccharides and their $\mathrm{N}$-acetyl groups by putative subsites of chitin deacetylase from a deuteromycete, Colletotrichum lindemuthianum. Biochemistry, 39, 8837-8843.

[25] Tsigos, I., Martinou, A, Kafetzopoulos, D., Bouriotis, V., (2000). Chitin deacetylases: new, versatile tools in biotechnology. Trends Biotechnol., 18, $305-312$.

[26] Ueno H, Mori T, Fujinaga T., (2001). Topical formulations and wound healing applications of chitosan. Adv Drug Deliv Rev., 52, 105-15.

[27] Vårum, K.M.; Smidsrød, O., (2005). Structure-property relationship in chitosans. In Polysaccharides: Structural Diversity and Functional Versatility; Dumitriu, S., Ed. Marcel Dekker: New York, NY, USA, , 625-642.

[28] Xing R, Liu S, Guo Z, Yu H., Wang P., Li C., Li Z., Li P., (2005). Relevance of molecular weight of chitosan and its derivatives and their antioxidant activities in vitro, Bioorg Med Chem, 13(5), 1573-1577. 
[29] You, Y., Park, W.H., Ko, B.M., Min, B.M., (2004). Effects of PVA sponge containing chitooligosaccharide in the early stage of wound healing. J. Mater. Sci.: Mater.Med., 15, 297-301. 